University of Nebraska - Lincoln

DigitalCommons@University of Nebraska - Lincoln

\title{
Redescription and Immature Stages of Promalactis Suzukiella (Matsumura) (Gelechioidea: Oecophoridae), A New Introduction into the United States
}

\author{
David Adamski \\ Department of Entomology, National Museum of Natural History, Smithsonian Institution, P.O. Box 37012, \\ MRC 168, Washington, D.C., 20013- 7012, U.S.A., adamskid@si.edu \\ Gary F. Hevel \\ Department of Entomology, National Museum of Natural History, P.O. Box 37012, NHB - CE716, \\ Smithsonian Institution, Washington, D.C. 20013-7012, hevelg@si.edu
}

Alan Pultyniewicz

6148 Agail Place, Columbia, MD 21045

Follow this and additional works at: https://digitalcommons.unl.edu/systentomologyusda

Part of the Entomology Commons

Adamski, David; Hevel, Gary F.; and Pultyniewicz, Alan, "Redescription and Immature Stages of Promalactis Suzukiella (Matsumura) (Gelechioidea: Oecophoridae), A New Introduction into the United States" (2009). USDA Systematic Entomology Laboratory. 51.

https://digitalcommons.unl.edu/systentomologyusda/51

This Article is brought to you for free and open access by the Entomology Collections, Miscellaneous at DigitalCommons@University of Nebraska - Lincoln. It has been accepted for inclusion in USDA Systematic Entomology Laboratory by an authorized administrator of DigitalCommons@University of Nebraska - Lincoln. 


\title{
REDESCRIPTION AND IMMATURE STAGES OF PROMALACTIS SUZUKIELLA (MATSUMURA) (GELECHIOIDEA: OECOPHORIDAE), A NEW INTRODUCTION INTO THE UNITED STATES
}

\author{
David Adamski, Gary F. Hevel, and Alan Pultyniewicz
}

(DA) Department of Entomology, National Museum of Natural History, P.O. Box 37012, NHB - E523, Smithsonian Institution, Washington, D.C. 20013-7012 (e-mail: david.damski@ars.usda.gov); (GFH) Department of Entomology, National Museum of Natural History, P.O. Box 37012, NHB - CE716, Smithsonian Institution, Washington, D.C. 20013-7012 (e-mail: hevelg@si.edu); (AP) 6148 Agail Place, Columbia, MD 21045

Abstract.-Promalactis suzukiella (Matsumura) was discovered independently several times over the past 5 years throughout the northeastern United States by private collectors and biophotographers. These discoveries represent the first records of this species in the United States and validate its approximate distributional range. Color photographs and a redescription of the adult, including the male and female genitalia, are provided. The larva and pupa of Promalactis are described in detail for the first time, with scanning electron micrographs and chaetotaxal maps. A lectotype for Borkhausenia suzukiella Matsumura, 1931, is designated herein. The importance of "backyard collecting" and amateur biophotography is emphasized, and participants are encouraged to continue the documentation of their findings through meetings, publication, and the internet.

Key Words: Asia, invasive species, Japan, rotting wood, tree bark

Species of Promalactis are small moths, usually brilliantly patterned with brownish-orange ground color scales, narrow white wing bands, small marginal patches or spots, and occasionally with some metallic or shiny scaled areas. There are about sixty species known worldwide, and they are found in the Eastern Palearctic (Lvovsky 1976, 1985, 1986a, 1986b) and Indo-Asian regions, from India to Far Eastern Asia (Meyrick 1908a, 1908b, 1922, 1931, 1935), including China, Korea (Park 1980, 1981; Park and Park 1998), and Japan (Matsumura 1931, Inoue 1954, Issiki 1957, Kuroko

* Accepted by David R. Smith
1959, Moriuti 1982). Larvae are known to feed under bark of rotting logs.

The purpose of this study is to document Promalactis suzukiella (Matsumura) (Oecophoridae) as a new introduction in the United States, provide evidence that it is established throughout many of the north Atlantic states along the east coast, and present details on its biology that are similar to those of its Asian congeners.

Evidence leading to the identity of the moth, Promalactis suzukiella, and its biology came from studies of Michael Gates and Mark Metz of the United States Department of Agriculture, Agricultural Research Service, Systematic 
Entomology Laboratory, National Museum of Natural History, Smithsonian Institution, Washington, D.C. A specimen of Promalactis suzukiella was reared while trying to obtain specimens of Balcha indica (Mani \& Kaul) (Eupelmidae), a parasitoid wasp of the emerald ash borer, Agrilus planipennis Fairmaire (Buprestidae), and specimens of Endomychus flavipes Ashmead (Pteromalidae), a parasitoid wasp of Endomychus biguttatus Say (Endomychidae), from rotting logs. Although the moth's identity was not known at the time, the site $\left(39^{\circ} 06^{\prime} 30^{\prime \prime} \mathrm{N}, 77^{\circ} 01^{\prime} 30^{\prime \prime} \mathrm{W}\right)$ with the $\log$ that Gates obtained had a large woodpile, which was examined later for lepidopterous larvae by the authors.

Approximately sixty larvae were discovered under bark of rotting logs. Larvae were collected using soft forceps or a camel's hair brush and placed into diet cups partially filled with a standard sugarcane borer diet (Southland Products ${ }^{\circledR}$, Lake Village, AR). Representative larvae and pupae were boiled in $\mathrm{H}_{2} \mathrm{O}$ and preserved in $70 \% \mathrm{EtOH}$ for later examination. The remaining pupae were allowed to complete development. Newly emerged adults were frozen and later spread and double-mounted. Only adults of Promalactis suzukiella were obtained from the rearing efforts.

For SEM study, larvae and pupae were cleaned in a full-strength solution of allpurpose cleaner, Formula 409 III ) detergent, and subsequently dehydrated in increasing concentrations of alcohol to absolute alcohol. After dehydration, specimens were critical point dried using a Tousimis critical point dryer, mounted on SEM stubs, and coated with gold-palladium (40/60\%), using a Cressington sputter coater. The ultrastructure of the larva and pupa was studied with an Amray 1810 scanning electron microscope at an accelerating voltage of $10 \mathrm{kV}$.

Gross morphological observations and measurements of larvae and pupae were made using a dissecting microscope (reflected light) with a calibrated micrometer. Genitalia were dissected as described by Clarke (1941), except Mercurochrome and chlorazol black were used as stains. The Methuen Handbook of Colour (Kornerup and Wanscher 1978) was used as a color standard. All voucher specimens of adults and immature stages generated from this study are deposited in the National Museum of Natural History, Smithsonian Institution, Washington, D.C. (USNM).

\section{Promalactis suzukiella (Matsumura 1931)}

(Figs. 1-18)

Diagnosis.-In the United States, Promalactis suzukiella may be confused with Callima argenticinctella Clemens (= Epicallima) due to similarities in size and maculation of the forewings. However, $P$. suzukiella is distinguished by having a white frons of the head and forewings with broader white basal and submedian bands, a larger white costal patch near the distal 2/3, and a conspicuous brownish-orange ground color.

Redescription.-Head: Vertex pale brownish orange; frontoclypeus white; scape of antenna white, flagellum with alternating black and white bands on each flagellomere; outer surface of labial palpus with segments 1-2 pale brownish orange, segment 3 brown with white apex, inner surface as above but paler; proboscis white. Thorax: Tegula and mesonotum pale brownish orange or brown on anterior $1 / 3$, pale brownish orange on posterior $2 / 3$. Foreleg with coxa and femur gray, tibia gray with 3 white spots (equally spaced) on anterior surface, tarsomere-1 white, tarsomere-2 gray, tarsomere-3 white, tarsomeres 4-5 black; midleg with femur pale yellowish gray, tibia gray with a narrow subapical band and apical band, spurs gray; tarsomeres as above; hindleg with femur 


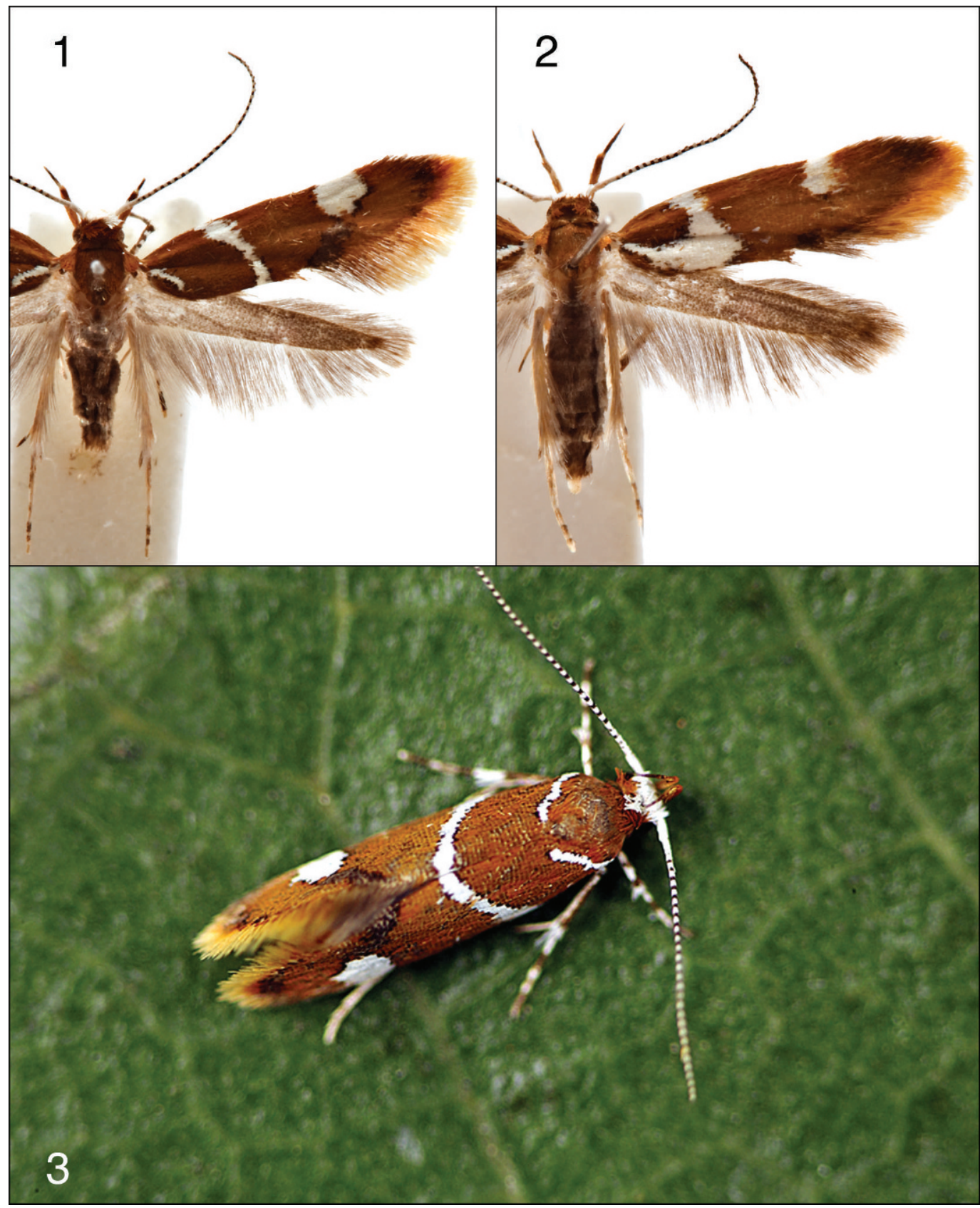

Figs. 1-3. Adults of Promalactis suzukiella (Matsumura). 1, A female from Prince Georges County, Maryland. 2, A female from same locality as above, showing variation in size of costal spot and fusing of basal and submedian bands. 3, Live adult on leaf.

white, tibia pale gray intermixed with pale brownish-orange scales, tarsomeres 1-2 pale gray intermixed with pale brownish-orange scales, tarsomeres 3-5 white. Forewing (Figs. 1-3) Length 4.5$7.0 \mathrm{~mm}(\mathrm{n}=161)$, brownish orange with a short, narrow, oblique band from base of $\mathrm{CuP}$ to $1 / 2$ distance from margin; 


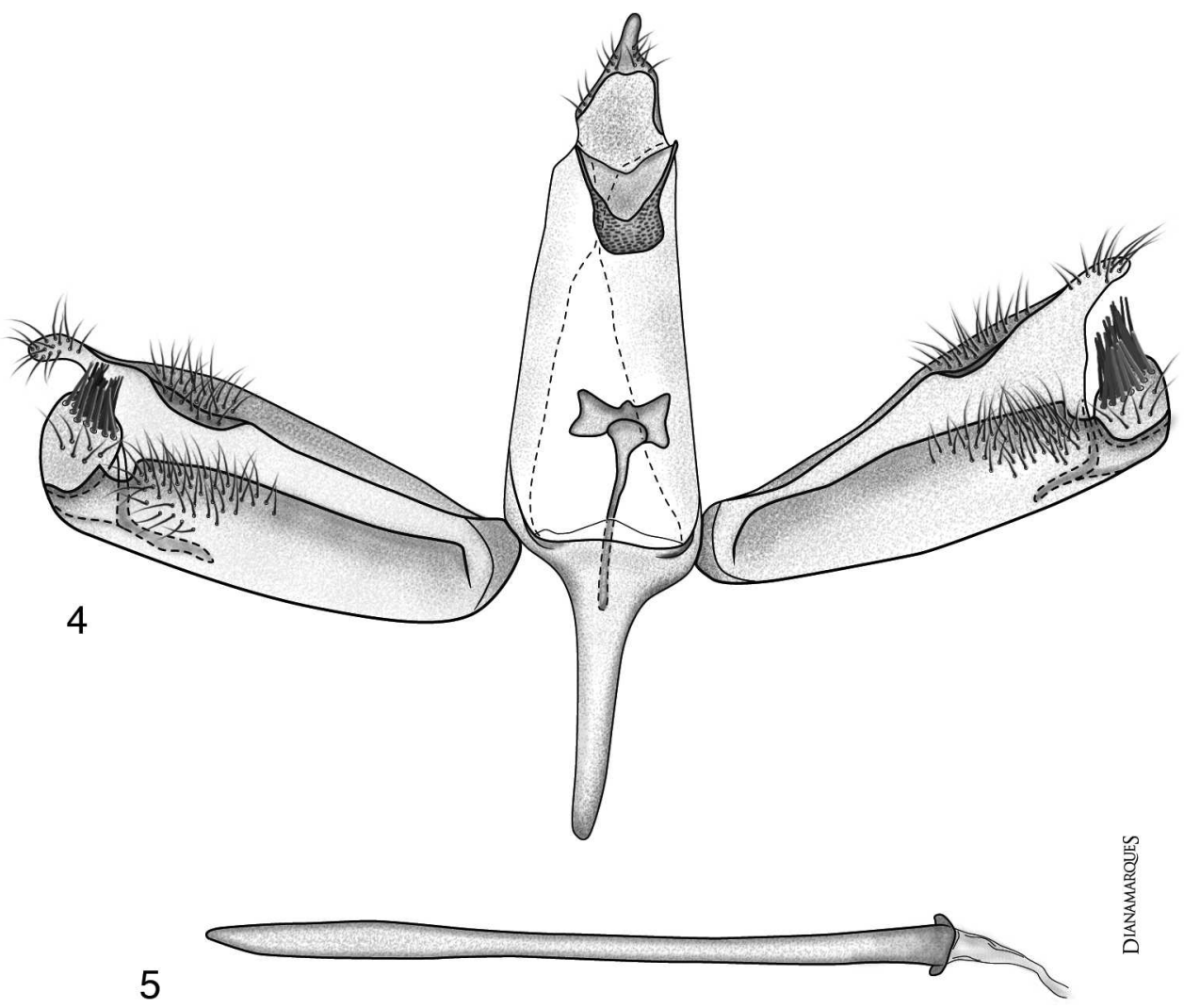

Figs. 4-5. Male genitalia of Promalactis suzukiella (Matsummura). 4, Genital capsule. 5, Aedeagus.

submedian oblique band white, wider anteriorly, slightly curved inwardly on posterior $1 / 2$; or rarely, basal band may be fused posteriorly with submedian band, forming a chevron-shaped marking (Fig. 2); a large white costal spot on distal $2 / 3$ connecting posteriorly with a recurrent shiny, brown band; few to several small, shiny brown spots along submargin; outer fringe pale brownish orange. Undersurface brownish gray. Hindwing pale gray near base, gradually darkening to apex. Abdomen (Figs. 1-2): Pale gray intermixed with pale-brown scales dorsally, white ventrally. Male genitalia (Figs. 4-5) with tegumen elongate and narrow, no wider than broadest part of valva; uncus hemispheric, open ventrally, with a short, spinelike apical process; gnathos dorsoventrally flattened, protuberant, wide, slightly con- stricted subapically; vinculum with elongate saccus; juxta a rodlike support with two short, lateral arms apically; valva narrow basally, gradually widened distally; costa with a small, setose, downcurved lobe near 2/3 length, and a short, setose, digitate apical process; outer margin broadly scalloped inwardly; ventral part of valva acutely upturned inwardly to beyond middle, with inner margin setose mesially, distoventral margin extending dorsolaterally, forming a slightly dorsoventrally flattened, cuplike process with a dense cluster of several spinelike setae; aedeagus narrow and elongate, longer than valva, narrowly rounded apically, slightly dilated basally. Female genitalia (Fig. 6) with papillae anales slightly divergent apically; apophyses posteriores about twice length of apophyses anteriores; lamella postvagi- 


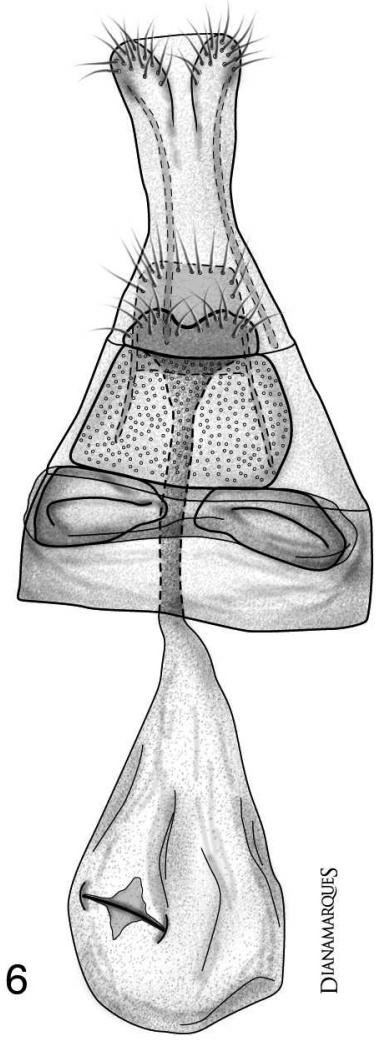

Fig. 6. Female genitalia of Promalactis suzukiella (Matsumura).

nalis broadly notched mesially forming two lateral and setose lobes; lamella antevaginalis broad; antrum slightly broadened posteriorly; ductus bursae extending beyond sixth sternum to corpus bursae; two broadly rounded, shallowly invaginated bullae between lamella antevaginalis and sixth sternum; corpus bursae with a platelike signum with a narrowly raised, inwardly directed transverse ridge.

Larva (Figs. 7-16). General: Length 7.6-11.6 mm $(\mathrm{n}=20)$. Body pale gray, surface texture with microconvolutions (seen using high magnification); most pinacula darker than body, setal sockets wide, demarcated by a circular rim; head capsule, prothoracic shield, thoracic legs, anal plate, and large pinaculum on proleg of A10 amber. Head (Figs. 7-10,
16): Hypognathous; epicranial suture absent, adfrontal sclerites meeting at base of epicranial notch; notch moderately deep forming two adjacent epicranial hemispheres; outer integument shallowly ridged with irregular polygons; AF2 slightly below apex of frons, at least twice length of AF1; F1 at least twice length as AF2, nearly in line with $\mathrm{C} 1, \mathrm{C} 2$ ventrolateral to $\mathrm{C} 1$; $\mathrm{P} 1$ in line with AF2 or slightly below, at least twice length of P2; P2 slightly dorsolateral to $\mathrm{P} 1$; L1 about half length of and posterolateral to A3; A3 about equal in length with and in line with A1; A2 in line with or slightly below F1, about half distance closer to A1 than to margin of adfrontal sclerite; six stemmata in an irregular Cshaped pattern, with stemmata 3-5 approximate; S3 posterolateral to stemma $-1, \mathrm{~S} 2$ equidistant to and posterior to stemmata 2-3; S1 slightly anteroventral to stemma - 1; SS3 below S1; SS2 closer to SS3 than to SS1; SS1 posterior to mandibular condyle; mandible with two subequal setae, and two prominant dentitions of five; anteclypeal ridge with a broadly rounded lobe between antennal base and mandible; labrum with six pairs of setae, two subequal median pairs, two subequal frontomarginal pairs, two subequal lateromarginal pairs; antennal sensilla as figured (Fig. 9); sensilla of maxillary palpus as figured (Fig. 10); spinneret short. Thorax (Figs. 13): T1 with L-group trisetose, L1 closer to L3 than to L2, on anterior part of pinaculum; pinaculum extending slightly beneath spiracle; L1 about twice length of L2, about five times longer than L3; $\mathrm{SV}$-group bisetose, on anterior part of pinaculum, SV1 about 1/3 longer than SV2; mesal margins of coxae nearly touching; V1s close, anterior to posterior margins of coxae (not shown); shield with SD1 slightly longer than XD2 and $\mathrm{XD} 1$, closer to SD2 than to XD2; SD2 short, anterior to D2 and D1; D2 longest seta on shield, slightly closer to D1 than 

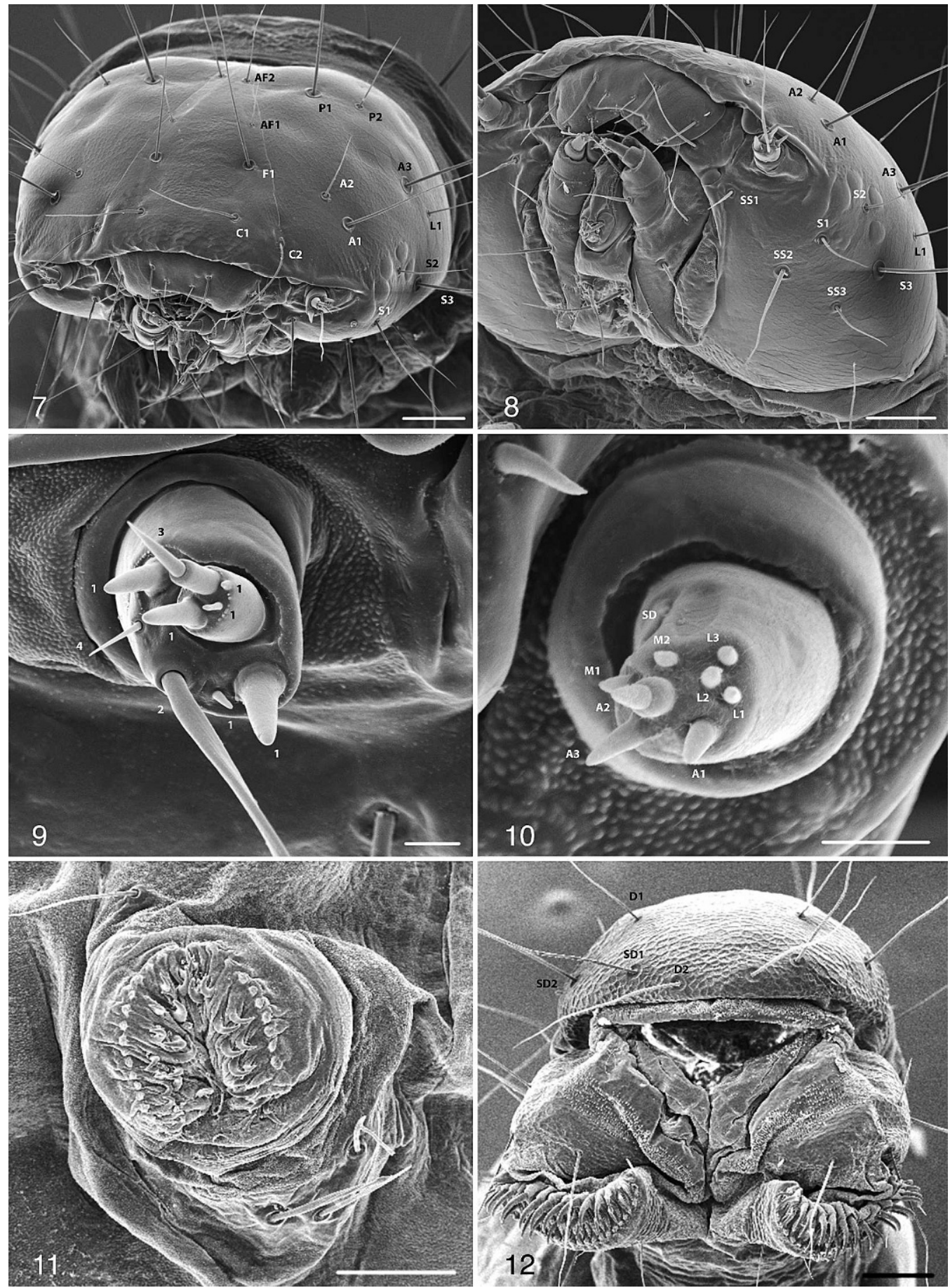

Figs. 7-12. Scanning electron micrographs of larva of Promalactis suzukiella (Matsumura). 7, Head (frontal view). Scale $=100 \mu .8$, Head (ventrolateral view). Scale $=100 \mu .9$, Right antenna and associated sensilla (apical view); $1=$ sensilla basiconica, $2=$ sensilla chaetica, $3=$ sensillum styloconicum, $4=$ sensillum trichodeum. Scale $=10 \mu$. 10, Left maxillary palpus and associated sensilla; A2 = sensillum styloconicum, A1, A3, M1, M2, L1, L2, and L3 = sensilla basiconica, SD = sensillum digitiform. Scale = $10 \mu$. 11, Proleg. Scale $=100 \mu .12$, Setae on anal plate of A10. Scale $=100 \mu$. 

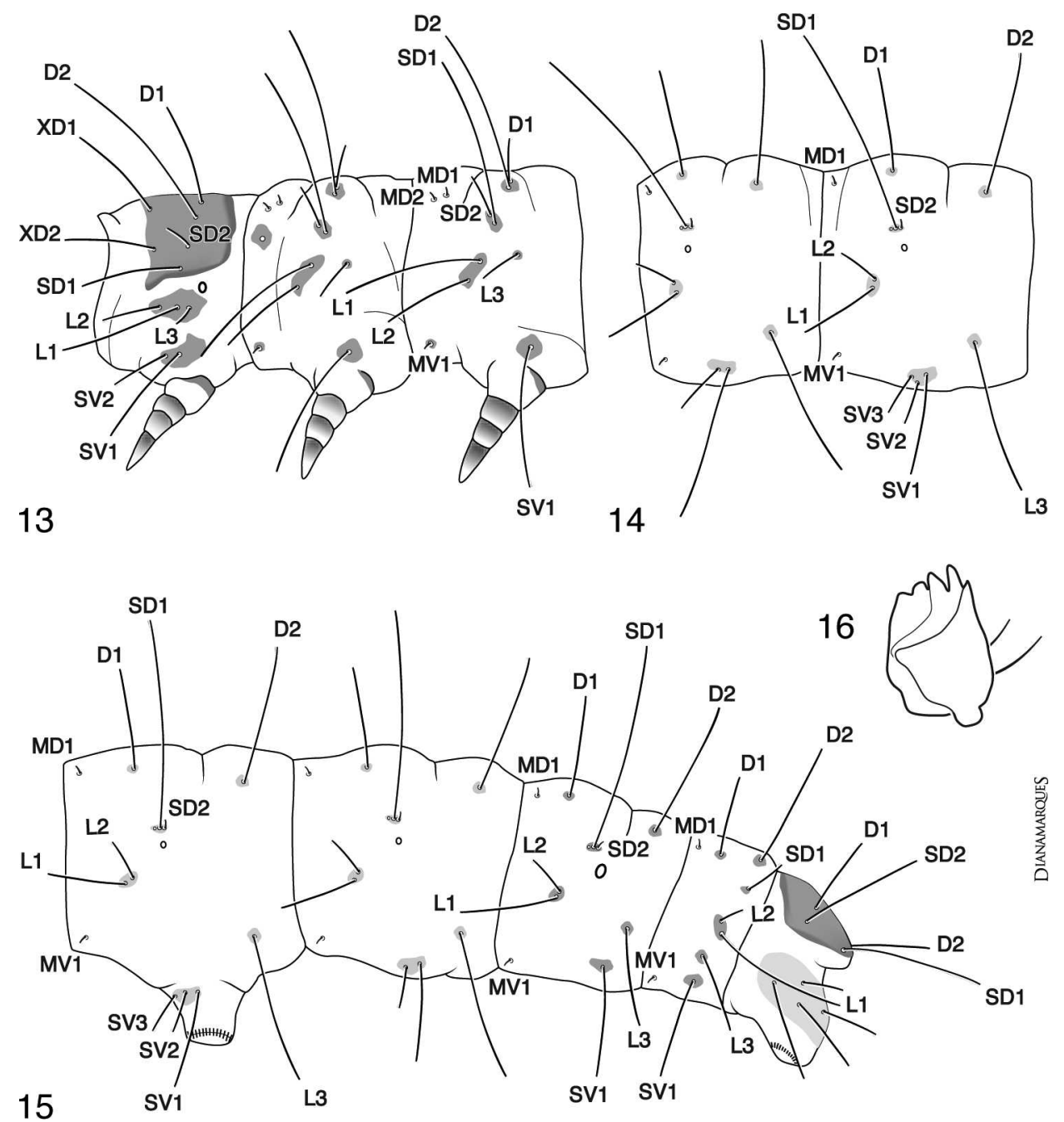

Figs. 13-16. Chaetotaxal maps of thorax and abdomen, and mandible of Promalactis suzukiella (Matsumura). 13, T1-T3 (lateral view). 14, A1-A2 (lateral view). 15, A6-A10 (lateral view). 16, mandible.

to SD2, nearly equidistant from XD2 and XD1; D1 slightly closer to median axis than XD1. T2-T3 (Fig. 13): D1 about 5 times longer than D2, on same pinaculum; SD1 about five times longer than SD2, on same pinaculum, anterior to D-group; MD1 and MD2 on anterior part of segment between D-group and SD-group; a large pinaculum (lacking seta) beneath MD-group slightly beneath SD-group pinaculum only on T2; L1 slightly longer than L2, on same pinaculum anterior to SD-group; L3 about 1/2 of L2, in horizontal line with or slightly above L1, and anterior to SV1; MV1 on anterior part of segment slightly above SV1; V1 3-4 times farther apart than V1s on $\mathrm{T} 1$, both pairs anterior to posterior margin of coxae (not shown). Abdomen: A1-A2 (Fig. 14) with D2 slightly longer than and lateral to D1; MD1 on anterior part of segment slightly below D1; SD1 dorsoanterior to spiracle on A1 and slightly more anterior on A2; SD2 minute, on same pinaculum posterior to SD1, a small pore on ventroanterior part of pinaculum; L1 slightly longer than L2, on same pinaculum, anterior to SD- 

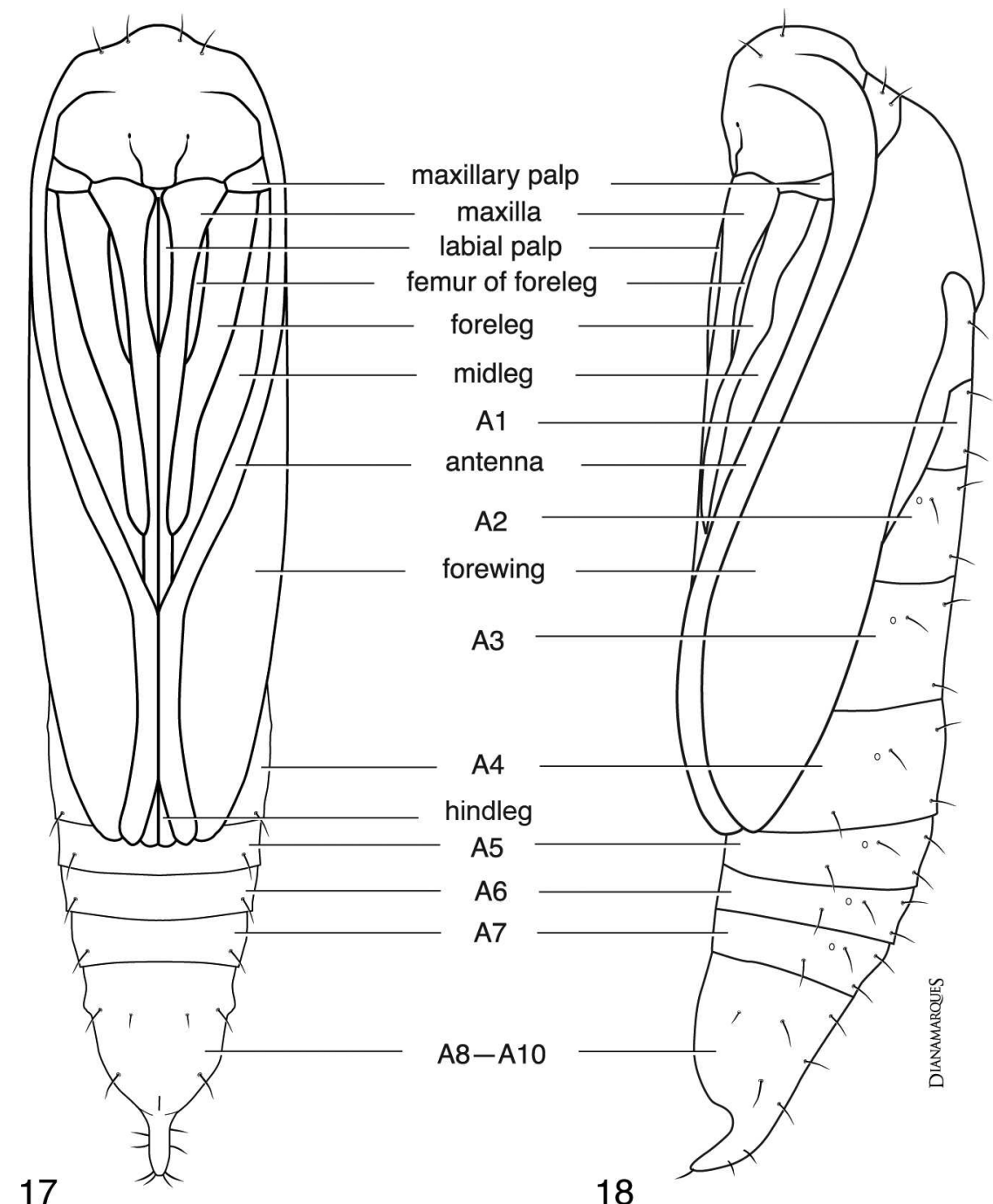

Figs. 17-18. Pupa of Promalactis suzukiella (Matsumura). 17, ventral view. 18, lateral view.

group; L3 about twice length of L1, anterior to, in horizontal line with, or posterior to D2; SV-group bisetose on A1, trisetose on A2; MV1 on anterior part of segment, even with or slightly below SV-group; V1s equidistant to V1s on T1-T2 and A3-A8 (not shown). A3A6 (Figs. 12-15) with 4 pairs of protuberant prolegs, crochets biordinal, in a circle, gradually shortened, uniordinal laterally; setae as above except SV-group trisetose. A7 as above except, SV-group setae bisetose. A8 with SV-group uni- setose; L3 slightly anterior to or in vertical line with D2; SV1 slightly anterior to L3, in line with V1. A9 with D1 ventroanterior to D2, with other setae nearly in a vertical line; MD1 and MV1 on anterior part of segment. A10 (Figs. 12, 15): anal plate with SD2 about 4-5 times farther from SD1 than from D2; D1 slightly posterior to SD1; prolegs bearing 27-30 biordinal crochets in a slight arc.

Pupa (Figs. 17-18): Length 4.2-5.2 (n $=6$ ). Amber, smooth, subcylindrical; 
spiracles protuberant. Sclerites of antennae widely separated anteriorly, gradually convergent from a point beyond and lateral to apical part of sclerites of femur of forelegs, fused a short distance beyond sclerites of maxillae, gradually divergent posteriorly, exposing distal part of sclerites of hindlegs; A5-A6 movable anteriorly and posteriorly, A7-A10 fused, movable as unit; dorsum of A10 bearing a large, cylindrical, ventrally curved, spinelike process.

Host.-In the United States, larvae of Promalactis suzukiella were found under bark of rotting logs of chokecherry, Prunus virginiana L. (Rosaceae), and oak, Quercus sp. (Fagaceae). In Asia, larvae were collected under bark of peach, Prunus persica (L.) (Rosaceae), especially among parts damaged by Synathedon (= Conopia) hector Butler (Sesiidae) (Park 1981).

Flight period.-Specimens of Promalactis suzukiella have been taken in March through to late September indicating that this species is at least bivoltine, and may be multivoltine.

Parasitoids.-No parasitoids were reared from over sixty larvae collected.

Distribution.-Promalactis suzukiella is known from Korea (Park 1981, Park and Park 1998), Japan, and Taiwan (Fujisawa 2002). In the United States, the first known specimens of $P$. suzukiella were collected by J. Glaser of Towson, Maryland in August of 2002. Subsequently, other regional entomologists collected P. suzukiella in Ashton, Bear Island (Montgomery County), Cheverly (Prince George's County), Scientist Cliffs (Calvert County), and Wittman (Talbot County), Maryland, and in Fairfax City, Great Falls National Park, and Turkey Run National Park (Fairfax County), Virginia. Presently, we know from biophotographers, that $P$. suzukiella is known from Cheverly and Bowie (Prince George's County), Maryland (Johnson 2006), Oakton (Fairfax Coun- ty), Virginia, central and northern New Jersey (no counties given), Brooklyn (Kings County), New York (Czaplak et al. 2007, Gould 2006, Line 2007), and Mountville (Lancaster County), Pennsylvania (Roth 2006). This insect is undoubtedly more widespread than reported herein. It is unknown how and when $P$. suzukiella became established and whether its introduction into the United States was due to a single founder event or from several such events from different ports of entry.

Type examined.-Lectotype + , designated herein, "Borkhausenia suzukiella M. [handwritten], det. Matsumura"; "Kyoto, [illegible]"; "ㅇ Genitalia on slide no. O[e]c[o]p[horidae]. 7, T. Kumata, 1940"; "Lectotype, Borkhausenia suzukiella Matsumura" [red handwritten label]; "Type Matsumura" [red label]. Although a female specimen bears a lectotype label, it has not been designated previously. The lectotype is located in the Entomological Institute, Hokkaido University, Sapporo, Japan.

Other specimens examined.-Twentythree specimens ( $3 \$ 20$ q) of Promalactis suzukiella from Japan were examined at HUS and 3 specimens at the National Museum of Natural History, Department of Entomology, Smithsonian Institution, Washington, D.C., USA. In addition, over 160 specimens of P. suzukiella collected in the District of Columbia region (Maryland and Virginia) by regional collectors were obtained for examination for this study and are deposited in USNM.

\section{Discussion}

The results of this study are solely dependent upon the shared information acquired serendipitously by professional biologists, amateur moth collectors and biophotographers. The information from Gates and Metz initially lead the authors to the back yard of the home of Gary Hevel, the site from which the logs that 
Gates acquired for the rearing of target parasitoids.

Once the reared moths from the Hevel residence were recognized as the same species collected by Gates and Metz, a search for the identity of the moth began. After confirmation of the species identity of the moth, distributional information from several "backyard" collectors was made available, in addition to vital distributional information from biophotographers.

As a testament to the importance of "backyard collecting," a four-year insect survey at the Hevel Residence (second author), approximately 2-acre plot, yielded more than 4,000 species of insects. Of these, two genera and four species have been recognized authoritatively as new to science, while others currently are being used in ongoing taxonomic research programs.

In retrospect, we could not have documented the biology, described the immature stages, obtained distributional records, or confirmed the identity of Promalactis suzukiella without open communication among professional biologists and enthusiastic amateurs. In this example, the sharing of information lead to a discovery that would not have occurred. We therefore strongly recommend that all professional biologists, "backyard" collectors, and biophotographers continue to use all forms of communication available; e-mail, meetings, publication, and the internet in order that bioinformation be shared and used by all.

\section{ACKNOWLedgments}

We thank Scott Whittaker, Manager, Scanning Electron Microscopy Laboratory, Smithsonian Institution, Washington, D.C., USA, for his suggestions on fixation of larvae and scheduling of SEM time; Toshiya Hirowatari, Entomological Laboratory, Graduate School of Agriculture and Biological Sciences,
Osaka Prefecture University, Sakai, Osaka, Japan, for access to the microlepidoptera collection; Sugisima Kazuhiro, Entomological Institute, Hokkaido University, Sapporo, Japan, for providing images of the lectotype of Promalactis suzukiella; Brent Steury, United States National Park Service, who facilitated insect surveys at sites along the George Washington Memorial Parkway in Virginia; and Diana Marques, scientific illustrator, Lisboa, Portugal, for the illustrations of the male and female genitalia, chaetotaxal maps of the larva and pupa, and the preparation of all the plates. We also thank John W. Brown, Michael Gates, John Glaser, Jean-François Landry, Mark Metz, Robert Patterson, Warren Steiner, and J.M. Swearingen for allowing us to examine their collections of Promalactis suzukiella. Finally, we thank the following biophotographers, Dave Czaplak, Anita Gould, Bill Johnson, Larry Line, Arlene Ripley, and Lynn Scott for making their images and distribution data accessible through the internet.

\section{Literature Cited}

Clarke, J. F. G. 1941. The preparation of slides of the genitalia of Lepidoptera. Bulletin of the Brooklyn Entomological Society 36: 149161.

Czaplak, D., L. Line, A. Ripley, and L. Scott. 2007. Moth Photographers Group. http:// mothphotographersgroup.msstate.edu/Files/Live/ BP/MothTalk/MothTalk022.htm (accessed 7 November 2007).

Fujisawa, K. 2002. The genus Promalactis (Oecophoridae) from Japan. Journal of the Japanese Heterocerists' 218: 337-350.

Gould, A. 2006. Images of Promalactis suzukiella, 1046.99, IMG_3946_edited. http:// flickr.com/photos/anitagould/251980794/in/ set-72157594193524242/ (accessed 7 November 2007).

Inoue, H. 1954. Check List of the Lepidoptera of Japan, Part I. Tokyo. pp. 62-63.

Issiki, S. 1957. Family Oecophoride, pp. 49-52, pls. 8, pp. 236-238. In Esaki, et al. eds. Icones Heterocerorum Japonicorum in Coloribus Naturalibus. Hoikusya, Osaka. 
Johnson, B. 2006. BugGuide, Introduction, Images, and Information for Insects, Spiders, and their Kin for the United States and Canada. Promalactis suzukiella photo \# 26501. http:// bugguide.net/node/view/62501/bgimage (Accessed 7 November 2007).

Kornerup, A. and J. H. Wanscher. 1978. Methuen Handbook of Colour. 2nd ed. Methuen and Co., Ltd., London. 243 pp.

Kuroko, H. 1959. Notes on the nomenclature of some Microlepidoptera in Japan. Ty $\square$ To Ga Lepidopterological Society of Japan 10(3): 34-35.

Line, L. 2007. Moths of Maryland and Specimens from other States. http://www.marylandmoths. com/ (Accessed 7 November 2007).

Lvovsky, A. 1976. Some little known species of Far Eastern Oecophorids (Lepidoptera: Oecophoridae). Trudy Zoologicheskogo Instituta, Leningrad 67: 56-59.

. 1985. New species of the broadwinged moth (Lepidoptera: Oecophoridae) from Primorye Region. Trudy Zoologichekogo Instituta, Leningrad 134: 95-101.

1986a. New species of broad-winged moths of the genus Promalactis Meyrick (Lepidoptera: Oecophoridae) of the USSR Far East, pp. 37-41. In Ler, P. ed. Systematics and Ecology of Lepidoptera from the Far east of the USSR. 1986b. A review of the broad-winged moths (Lepidoptera: Oecophoridae) of the Far East. Trudy Zoologicheskogo Instituta, Leningrad 145: 72-74.
Matsumura, S. 1931. 6000 Illustrations. Insects Japan-Empire. Tokyo. pp. 1088-1089.

Meyrick, E. 1908a. Descriptions of Indian microlepidoptera. Journal of Bombay Natural History Society 15: 806-812.

. 1908b. New micro-lepidoptera from India and Burma. Records of the Indian Museum 2: 395-400.

. 1922. Family Oecophoridae. In Wytsman, P. ed. Genera Insectorum, Brussels. 240 pp.

1931. The Pyralidae and micro-lepidoptera of Kwanshien. Bulletin de la Section Scientifique of the Académie Roumaine 14(3): 66-75.

. 1935. Exotic Micro-Lepidoptera. Vol. 4. Taylor and Francis, London. 642 pp.

Moriuti, S. 1982. Family Oecophoridae, pp. 251252. In Inoue, et al. eds. Moths of Japan, Part I, and p. 206; Part II, pl. 11. Tokyo.

Park, K. T. 1980. A new Promalactis species from Korea (Lepidoptera, Oecophoridae). The Korean Journal of Plant Protection 19(3): 145-147.

. 1981. A revision of the Genus Promalactis of Korea (Lep., Oecophoridae). The Korean Journal of Plant Protection 20(1): 43-50.

Park, K. T. and Y. M. Park. 1998. Genus Promalactis Meyrick (Lepidoptera: Oecophoridae) from Korea, with descriptions of six new species. Journal of Asia-Pacific Entomology 1(1): $51-70$.

Roth, M. 2006. Small moth - Epicallima. http:// bugguide.net/node/view/56605/bgimage (Accessed 4 March 2007) 